\title{
Local probing of multiferroics: First-principles study of hyperfine parameters in $\mathrm{YMnO}_{3}$ and $\mathrm{YMn}_{2} \mathrm{O}_{5}$
}

\author{
J. N. Gonçalves ${ }^{1 a}$, V. S. Amaral ${ }^{1}$, J. G. Correia ${ }^{2}$, A. Stroppa ${ }^{3}$, A. S. Fenta ${ }^{1}$, A. Baghizadeh ${ }^{4}$, S. Picozzi $^{3}$ \\ ${ }^{1}$ Departamento de Física and CICECO, 3810-093 Universidade de Aveiro, Portugal \\ ${ }^{2}$ Instituto Tecnológico e Nuclear, Instituto Superior Técnico, Universidade Técnica de Lisboa, 2686-953 Sacavém, Portugal \\ ${ }^{3}$ CNR-SPIN, 67100 L'Aquila, Italy \\ ${ }^{4}$ Departamento de Engenharia de Materiais e Cerâmica and CICECO, 3810-093 Universidade de Aveiro, Portugal
}

\begin{abstract}
We model the ferroelectric and paraelectric phases in the $\mathrm{YMnO}_{3}$ and $\mathrm{YMn}_{2} \mathrm{O}_{5}$, compounds with discussion of the hyperfine parameters at the atomic nuclei: electric field gradient and magnetic hyperfine field, using first-principles density functional theory FP-L/APW+lo method (WIEN2K code). The differences of the changes in hyperfine properties and their correlation due to the onset of polarization in both cases reveal their sensitivity to the different electronic densities changes due to ferroelectricity. In the case of $\mathrm{YMnO}_{3}$ the greater changes appear in the $\mathrm{Y}$ and $\mathrm{O}$ atoms, while in $\mathrm{YMn}_{2} \mathrm{O}_{5}$, where the polarization is induced by a magnetic transition, the parameters at $\mathrm{Mn}$ and its bonded $\mathrm{O}$ atoms are changed the most. The sensitivity of the parameters to different degrees of approximation in calculations is also discussed.
\end{abstract}

\section{Introduction}

The multiferroic materials in which magnetic and electric orders coexist simultaneously in a single phase have attracted immense interest from the fundamental and application fronts [1]. Ferroelectric and magnetically ordered compounds are especially interesting because of the potentially large coupling between electric (magnetic) fields and magnetization (polarization) [2,3]. Manganese oxides are one particular class of compounds very studied in this respect. The Mn atoms usually form frameworks with $\mathrm{Mn}$ centered polyhedra with $\mathrm{O}$ at the vertices, comprising octahedra or pyramids, as is the case in the orthorhombic E-type, hexagonal $\mathrm{RMnO}_{3}$ or in the $\mathrm{RMn}_{2} \mathrm{O}_{5}$ compounds ( $\mathrm{R}$ denoting rare-earths, $\mathrm{Y}$ or $\mathrm{Bi}$ ). $\mathrm{We}$ focus on the calculation of hyperfine quantities in $\mathrm{YMnO}_{3}$ and $\mathrm{YMn}_{2} \mathrm{O}_{5}$. These two compounds in particular comprise two different mechanisms of improper ferroelectricity, and we will show how this is reflected in the calculated electronic density features and particularly the hyperfine parameters: the electric field gradient (EFG), and magnetic hyperfine field (MHF).

Measurements of the hyperfine interactions between probe nuclei and their local environment are a useful complement to the more conventional measurements, providing information at the sub-nano (atomic) scale. In this respect, many experimental techniques can be used, such as perturbed angular correlations spectroscopy, nuclear magnetic resonance or Mössbauer effect spectroscopy. The interpretation of these data is not always straightforward and first-principles density functional theory calculations are the theoretical basis to complement the experiments. We focus on hyperfine EFG, and MHF, which are obtained from the selfconsistent electronic density calculation results.

The quadrupole electric interaction occurs between nuclear quadrupole moments (with nuclear spin higher than $1 / 2$ ) and their local electron environment, quantified by the EFG, the tensor of second derivatives, with respect to coordinates, of the Coulomb potential at the nuclear position. The traceless and diagonalized tensor components are usually named by their magnitude as $\left|\mathrm{V}_{\mathrm{zz}}\right| \geq\left|\mathrm{V}_{\mathrm{yy}}\right| \geq\left|\mathrm{V}_{\mathrm{xx}}\right|$, and the tensor may be characterized two parameters, the highest component $\mathrm{V}_{\mathrm{zz}}$ and the asymmetry $\eta=\left(V_{x x}-V_{y y}\right) / V_{z z}(0 \leq \eta \leq 1)$. It can be a finger print of structural, electronic properties, defects, and dynamical phenomena, and it can also exhibit some changes with magnetic order, since it is related to the electron density close to the nucleus [4].

The magnetic dipole interaction comes from the nuclear magnetic moments interacting with the magnetic field at the nucleus. The MHF is related to the local and induced moments at each site. It is characterized by three main contributions: Fermi contact term (spin electron density at the nucleus), and the on-site orbital and spin dipole moment interactions [5]. The on-site terms are calculated inside atomic spheres used in the calculation method. The contribution from the remaining regions in the lattice should be negligible, and will not be calculated in this work.

a Corresponding author: joaonsg@ua.pt 


\section{Calculation Details}

The L/APW+lo method is used, as implemented in WIEN2K [6] code. It is an accurate full potential all electron method, appropriate for the calculation of hyperfine parameters. We use the $\mathrm{LDA}+\mathrm{U}$ approximation $[7,8]$, with $\mathrm{U}_{\text {eff }}=\mathrm{U}-\mathrm{J}$ applied in the $\mathrm{Mn} \mathrm{d}$ orbitals equal to 7 and $6 \mathrm{eV}$, for $\mathrm{YMnO}_{3}$ and $\mathrm{YMn}_{2} \mathrm{O}_{5}$ respectively. Spin-orbit coupling is calculated from the scalar-relativistic result with a second-variational procedure, the magnetization along the [001] direction. We do not analyse the dependence of the parameters with the magnetization direction. We tested the number of $\mathbf{k}$ points and maximum wave number of the plane waves used in the calculations, in order to get converged values of the hyperfine parameters.

\section{Results and Discussion}

\section{1. $\mathrm{YMnO}_{3}$}

In the rare-earth hexagonal manganites $\mathrm{RMnO}_{3}$ the $\mathrm{Mn}$ atoms form two-dimensional triangular lattices, linked by oxygen atoms and separated by layers of rare-earth and oxygen atoms, forming $\mathrm{MnO}_{5}$ bi-pyramids between rareearth planes (figure 1). The ferroelectric phase ordering occurs at $\mathrm{T}_{\mathrm{C}} \sim 1000 \mathrm{~K}$ while the magnetic transition occurs at $\mathrm{T}_{\mathrm{N}} \sim 80 \mathrm{~K}$. The high temperature paraelectric phase and the ferroelectric phase have the space groups $P 63 / \mathrm{mmc}$ and $P 63 \mathrm{~cm}$, with symmetry loss in the ferroelectric phase associated to a tilting of the $\mathrm{MnO}_{5}$ bipyramids and a buckling of the rare-earth horizontal planes. $\mathrm{YMnO}_{3}$ is named a geometric ferroelectric, as ferroelectricity originates from the electrostatic interactions, without the involvement of hybridization changes [9], in distinction to more usual ferroelectrics.

For the calculations, the paraelectric [10] and ferroelectric [11] structures are taken from experimental data. We consider the ferromagnetic state for the paraelectric phase and an approximation to the frustrated magnetic state for the ferroelectric phase, a collinear magnetism where one $\mathrm{Mn}$ atom has opposite spin to the other $\mathrm{Mn}$ atoms in the same plane, following Medvedeva et al. [12] (figure 1). This state is $\sim 0.5 \mathrm{eV}$ lower in energy than the ferromagnetic state with the same structure. There are 20 different atoms due to the loss of magnetic symmetry, four Mn atoms, eight apical and four equatorial oxygen atoms. It is found, however, that this loss of symmetry nevertheless leaves pairs of atoms with approximately the same values of hyperfine parameters. The averages of the values for the ten pairs of atoms are presented. The results obtained for the electronic structure are consistent with previous first-principles calculations, with other methods $[12,13,14]$.

Figure 1. Paraelectric (left) and ferroelectric (right) structures of $\mathrm{YMnO}_{3}$, with the arrows representing the magnetic orders used in the calculations.

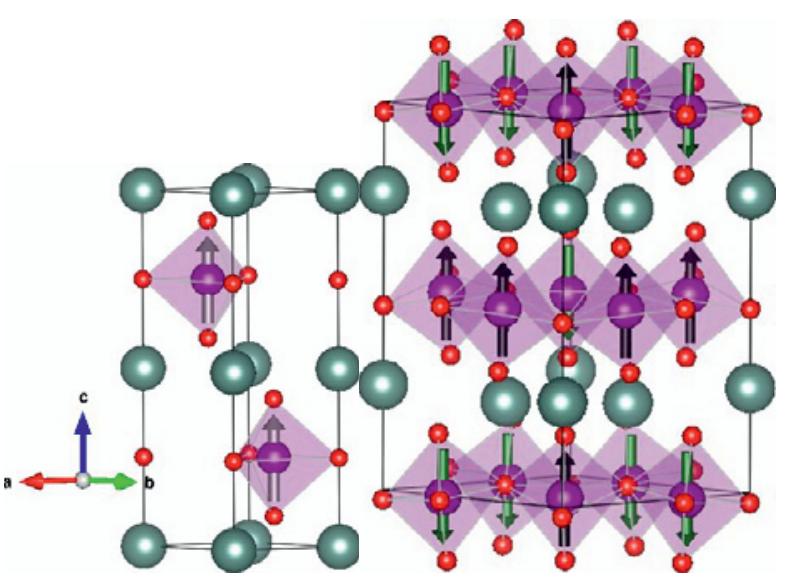

Table 1. Calculated $\mathrm{V}_{\mathrm{zz}}$ (units: $10^{21} \mathrm{Vm}^{-2}$ ) at the ferromagneticparaelectric and antiferromagnetic-ferroelectric phases of $\mathrm{YMnO}_{3}$, and the difference between both.

\begin{tabular}{|c|c|c|c|}
\hline Atom & FM-PE & AFM-FE & Difference \\
\hline & $\mathrm{V}_{\mathrm{zz}}$ & $\mathrm{V}_{\mathrm{zz}}$ & $\Delta \mathrm{V}_{\mathrm{zz}}$ \\
\hline $\mathrm{Y} 1$ & 10.68 & 0.44 & $\mathbf{1 0 . 2 4}$ \\
\hline $\mathrm{Y} 2$ & 10.68 & 6.12 & $\mathbf{4 . 5 6}$ \\
\hline $\mathrm{Mn} 1$ & -1.31 & -1.02 & -0.29 \\
\hline $\mathrm{Mn} 2$ & -1.31 & -0.84 & -0.47 \\
\hline $\mathrm{O} 1$ & 3.52 & 3.35 & 0.17 \\
\hline $\mathrm{O} 2$ & 3.52 & 3.33 & 0.19 \\
\hline O3 & 3.52 & 3.20 & 0.32 \\
\hline O4 & 3.52 & 3.21 & 0.31 \\
\hline O5 & -6.33 & -6.54 & 0.21 \\
\hline O6 & -6.33 & -5.85 & -0.48 \\
\hline
\end{tabular}

Table 2. Calculated MHF parameters (T) at the ferromagneticparaelectric and antiferromagnetic-ferroelectric phases of $\mathrm{YMnO}_{3}$.

\begin{tabular}{|c|c|c|c|c|c|}
\hline Atom & \multicolumn{2}{|c|}{ PE } & \multicolumn{2}{c|}{ FE } & \\
\hline & MHF & contact & MHF & contact & $\Delta|\mathrm{MHF}|$ \\
\hline Y1 & 5.01 & 4.91 & -1.01 & -0.95 & 6.02 \\
\hline Y2 & 5.01 & 4.91 & 0.79 & 0.77 & 4.22 \\
\hline Mn1 & -33.66 & -21.61 & -23.88 & -27.44 & 9.98 \\
\hline Mn2 & -33.66 & -21.61 & -27.35 & -30.83 & 6.31 \\
\hline O1 & -3.45 & -0.88 & 0.46 & -0.98 & 2.99 \\
\hline O2 & -3.45 & -0.88 & 0.47 & -0.94 & 2.98 \\
\hline O3 & -3.45 & -0.88 & 0.54 & -0.91 & 2.91 \\
\hline O4 & -3.45 & -0.88 & 0.58 & -0.84 & 2.87 \\
\hline O5 & 7.84 & 6.14 & 0.03 & 1.70 & 7.81 \\
\hline O6 & 7.84 & 6.14 & 2.26 & 1.67 & 5.58 \\
\hline
\end{tabular}

Table 1 presents $\mathrm{V}_{\mathrm{zz}}$ in the paraelectric and ferroelectric phases. In relation to the double pyramids, O1O4 are apical and O5-O6 are equatorial. There are small changes in the EFG at $\mathrm{Mn}$ and $\mathrm{O}$ atoms, but the larger changes are located at the $\mathrm{Y}$ atoms, in spite of the change in magnetic order. This is consistent with the larger displacements of $\mathrm{Y}$ atoms in the ferroelectric transition. 
Compared to the standard LDA calculations, it is found that the applied $U$ produces changes in EFG mainly at the $\mathrm{Mn}$ atoms, which have a decrease in absolute value relative to the simple LDA calculation ( $\sim-4.9$ in the LDA), and also small changes in the $\mathrm{O}$ atoms. Spin-orbit coupling introduced only negligible changes.

Table 2 presents the MHF, and its contact contribution, which is usually dominant. However, the additional dipolar hyperfine fields at $\mathrm{Mn}$ are particularly high, changing considerably the MHF $\left(\mathrm{B}_{\mathrm{dip}}=-11.6 \mathrm{~T}\right)$, and also at the oxygen atoms $(2.6 \mathrm{~T}$ and $1.7 \mathrm{~T}$ at apical and equatorial atoms resp.) in the paraelectric phase. The orbital fields are much smaller, being at most $-0.4 \mathrm{~T}$ at the $\mathrm{Mn}$ atoms. There are changes of the same order (3 to $10 \mathrm{~T})$ at all the atoms, with no particular changes higher in $\mathrm{Y}$ as in the EFG case. This is due to the change in magnetic order with consequent change in local and induced moments.

A different magnetic state (A-AF) was calculated (not shown) and it was found that the EFG changes very little, which is expected from the weak interlayer exchange, and the locality of the EFG. The MHF, apart from the changes of sign in half of the atoms, changes significantly only at $\mathrm{Y}$, where it becomes 0 .

\section{2 $\mathrm{YMn}_{2} \mathrm{O}_{5}$}

$\mathrm{YMn}_{2} \mathrm{O}_{5}$ is a multiferroic below $\mathrm{T}_{\mathrm{C}}=39 \mathrm{~K}$, where the polarization is induced by a change of magnetic order, from an incommensurate to a commensurate phase $[15,16]$. The $\mathrm{MnO}_{6}$ octahedra form infinite chains along one direction and share edges with other octahedra in the perpendicular plane. For the case of $\mathrm{YMn}_{2} \mathrm{O}_{5}$ we modelled the transition by using the ferromagnetic (paraelectric) and collinear uncompensated antiferromagnet also considered by Partzsch et al. [17]: an approximation to the true magnetic structure, by the splitting of the Mn atoms at the center of the pyramids (Mn2) into two groups with opposite spin polarizations. This results in additional splitting of $\mathrm{O} 2, \mathrm{O} 3$ and $\mathrm{O} 4$ atoms (figure 2). The resulting structure is in the $P m c 21$ space group. In this case the distortions due to ferroelectricity are small and not resolved experimentally, and we accordingly do not distort the structure and change only the spins.

Figure 2. Structure of $\mathrm{YMn}_{2} \mathrm{O}_{5}$ used in the calculations. In the antiferromagnetic structure, the $\mathrm{Mn} 2, \mathrm{O} 2, \mathrm{O} 3$ and $\mathrm{O} 4$ atoms are split in inequivalent atoms, due to the change of the $\mathrm{Mn} 2 \mathrm{~b}$ atom to an opposite spin. For simplicity, Y atoms are not shown.

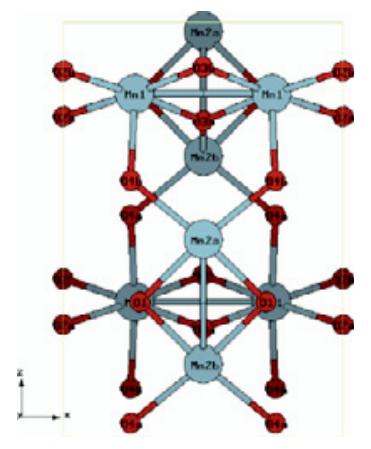

Table 3 presents the changes in the EFG parameters. Changes at $\mathrm{Y}$ sites are almost negligible, as expected due to the fact that they are far from the $\mathrm{Mn} 2 \mathrm{~b}$ atoms, where the spin is changed. Changes in EFG at Mn are not negligible at $\mathrm{Mn} 2 \mathrm{~b}$ and also $\mathrm{Mn} 1$ atoms, and interestingly the changes are even higher at some $\mathrm{O}$ atoms than at these two $\mathrm{Mn}$ atoms. In particular, the $\mathrm{O} 3$ atom has clearly the highest change of 1.3.

Table 3. Calculated EFG parameters $\left(\mathrm{V}_{\mathrm{zz}}\right.$ unit: $\left.10^{21} \mathrm{Vm}^{-2}\right)$ at the ferromagnetic-paraelectric and antiferromagnetic-ferroelectric phases of $\mathrm{YMn}_{2} \mathrm{O}_{5}$.

\begin{tabular}{|c|c|c|c|c|c|c|}
\hline Atom & \multicolumn{2}{|c|}{ F-PE } & \multicolumn{2}{c|}{ AF-FE } & \multicolumn{2}{c|}{ Difference } \\
\hline & $\mathrm{V}_{\mathrm{zz}}$ & $\eta$ & $\mathrm{V}_{\mathrm{zz}}$ & $\eta$ & $\Delta\left|\mathrm{V}_{\mathrm{zz}}\right|$ & $\Delta \eta$ \\
\hline $\mathrm{Y} 1$ & 5.59 & 0.25 & 5.51 & 0.25 & 0.08 & 0.00 \\
\hline $\mathrm{Y} 2$ & 5.59 & 0.25 & 5.49 & 0.22 & 0.09 & 0.03 \\
\hline $\mathrm{Mn} 1$ & 0.97 & 0.76 & -1.29 & 0.92 & 0.32 & -0.16 \\
\hline $\mathrm{Mn} 2 \mathrm{a}$ & 2.26 & 0.44 & 2.24 & 0.41 & 0.03 & 0.03 \\
\hline $\mathrm{Mn} 2 \mathrm{~b}$ & 2.26 & 0.44 & 1.94 & 0.50 & 0.33 & -0.07 \\
\hline O1 & -2.78 & 0.11 & -2.77 & 0.09 & -0.01 & 0.02 \\
\hline O2a & -4.32 & 0.60 & -4.39 & 0.53 & 0.07 & 0.07 \\
\hline O2b & -4.32 & 0.60 & -4.75 & 0.59 & 0.44 & 0.01 \\
\hline O3a & -8.93 & 0.12 & -7.65 & 0.03 & -1.27 & 0.08 \\
\hline O3b & -8.93 & 0.12 & -8.71 & 0.10 & -0.21 & 0.02 \\
\hline O4a & 4.73 & 0.68 & 4.69 & 0.60 & 0.04 & 0.08 \\
\hline O4b & 4.73 & 0.68 & 4.97 & 0.68 & -0.24 & -0.00 \\
\hline
\end{tabular}

Table 4. Calculated MHF parameters (T) at the ferromagneticparaelectric and antiferromagnetic-ferroelectric phases of $\mathrm{YMn}_{2} \mathrm{O}_{5}$.

\begin{tabular}{|c|c|c|c|c|c|}
\hline Atom & \multicolumn{2}{|c|}{ F-PE } & \multicolumn{2}{c|}{ AF-FE } & \\
\hline & MHF & contact & MHF & contact & $\Delta|\mathrm{MHF}|$ \\
\hline Y1 & 7.88 & 8.00 & 3.93 & 3.89 & 3.95 \\
\hline Y2 & 7.88 & 8.00 & 4.64 & 4.57 & 3.23 \\
\hline Mn1 & -16.06 & -15.66 & -16.97 & -16.91 & -0.91 \\
\hline Mn2a & -19.67 & -14.16 & -23.04 & -17.18 & -3.36 \\
\hline Mn2b & -19.67 & -14.16 & 27.23 & 20.76 & -7.56 \\
\hline O1 & -0.27 & 0.26 & 0.37 & 0.34 & -0.10 \\
\hline O2a & 3.53 & 1.16 & 3.49 & 1.23 & 0.03 \\
\hline O2b & 3.53 & 1.16 & 2.45 & 0.16 & 1.08 \\
\hline O3a & 12.04 & 9.02 & -3.78 & -5.99 & 8.23 \\
\hline O3b & 12.04 & 9.02 & 10.63 & 8.19 & 1.41 \\
\hline O4a & -1.70 & 0.34 & -0.97 & 0.84 & 0.73 \\
\hline O4b & -1.70 & 0.34 & -1.96 & 0.04 & -0.26 \\
\hline
\end{tabular}

Table 4 presents the MHF calculated in the same phases, and the difference between their absolute value. The contact contribution is also discriminated, and it dominates at $\mathrm{Y}$ and $\mathrm{Mn} 1$. However, at the other $\mathrm{Mn}$ and at $\mathrm{O}$ atoms, there are quite significant contributions from the orbital and dipolar contributions. Inspection of these contributions (not presented here) shows that the dipolar 
contribution is usually one order of magnitude higher than the orbital one $\left(\left|\mathrm{B}_{\text {dip }}\right|=5\right.$ and $2 \mathrm{~T}$ at $\mathrm{Mn}, 2 \mathrm{~T}$ at some $\mathrm{O}$ atoms. The difference of hyperfine fields is also dominantly due to the contact components, followed by the difference from the dipolar contributions.

The conspicuous shared $\mathrm{MnO}_{6}$-octahedral edges contain the $\mathrm{O} 3$ atoms with the higher EFGs and MHFs. These atoms are also special in other respect: whereas the other oxygen atoms in the structure have two Mn atoms as nearest neighbours, the atom forming the shared edges of the octahedra have three; the two Mn forming the octahedra and one Mn forming a pyramid. Since the spin of the $\mathrm{Mn}$ nearest neighbours is related to the hybridization with the $\mathrm{O} 3$ atoms, with the FM-AFM transition one of the $\mathrm{O} 3$ nearest neighbours changes to opposite spin, which causes changes in the surrounding electron density. These are reflected in the noticeable changes of $\mathrm{V}_{\mathrm{zz}}$ between the two cases, $\Delta \mathrm{V}_{\mathrm{zz}}$, which are indeed higher for the $\mathrm{O} 3$ atoms in relation to the other $\mathrm{O}$ atoms. In particular, they are higher for the $\mathrm{O} 3 \mathrm{a}$ atoms. The same thing happens for the hyperfine fields, presenting the highest change at the $\mathrm{O} 3 \mathrm{a}$ atom, among oxygen atoms. The $\mathrm{O} 3 \mathrm{a}$ atom is comparatively closer to the $\mathrm{Mn} 2 \mathrm{~b}$ atom, which changes spin direction, than $\mathrm{Mn} 2 \mathrm{a}$, consistent with the higher changes in O3a than in $\mathrm{O} 3 \mathrm{~b}$.

\section{Conclusions}

In $\mathrm{YMnO}_{3}$, the atoms showing the largest deviation in EFG going from paraelectric to ferroelectric structures are the $\mathrm{Y}$ atoms. This is consistent with the geometric nature of the ferroelectricity, due to the fact that the $\mathrm{Y}$ atoms have the largest displacements.

This contrasts with the spin-dependent hybridization in $\mathrm{YMn}_{2} \mathrm{O}_{5}$ [17], which results in ferroelectricity. A specific oxygen atom (O3a) plays a key role, and is associated with larger changes when passing from the ferromagnetic-paraelectric to the antiferromagneticferroelectric phase, in both EFGs and MHFs. It should be noted that the magnetic order is simplified in relation to experiment. Still, the results clearly illustrate the atomic scale resolving power of the hyperfine parameters $[18,19]$. In contrast with $\mathrm{YMnO}_{3}$, here the changes in the EFG are much smaller, only significant at the Mn and some $\mathrm{O}$ atoms, which is not surprising, considering, that there is a change of magnetic order while the structure remains the same. Summarizing, we showed how in two chemically similar multiferroic compounds the differences in hyperfine parameters give additional atomic specific information, consistent with ferroelectricity due to different origins. Their experimental investigation will be reported elsewhere.

\section{Acknowledgments}

This work was supported by project CERN/FP/123585/2011 (financed by FCT and
COMPETE/FEDER

program), PEstC/CTM/LA0011/2011 and PEstC/CTM/LA0011/2013. The FCT/CNR Bilateral agreement 2013/2014-1953 is also gratefully acknowledged. Authors acknowledge their FCT grants: SFRH/BPD/82059/2011 (JNG)，SRRH/BD/51140/2010 (AB) and SFRH/BD/84743/2012 (AF)

\section{References}

1. S. W. Cheong, M. Mostovoy, Nature Mater. 6, 13 (2007)

2. M. Fiebig, J. Phys. D: Appl. Phys. 38, R123 (2005)

3. T. Kimura, T. Goto, H. Shintani, K. Ishizaka, T. Arima, Y. Tokura, Nature 426, 55 (2003)

4. P. Blaha, K. Schwarz, P. Herzig, Phys. Rev. Lett. 54, 1192 (1985)

5. S. Blügel, H. Akai, R. Zeller, P. H. Dederichs, Phys. Rev. B 35, 3271 (1987)

6. P. Blaha, K. Schwarz, G. K. H. Madsen, D. Kvasnicka, and J. Luitz, WIEN2K: An Augmented Plane Wave+Local Orbitals Program for Calculating Crystal Properties (Karlheinz Schwarz, Technische Universität, Wien, Austria, 1999).

7. J. P. Perdew, Y. Wang, Phys. Rev. B 45, 13244 (1992)

8. V. I. Anisimov, I. V. Solovyev, M. A. Korotin, M. T. Czyżik, G. A. Sawatzky, Phys. Rev. B 48, 16929 (1993)

9. B. B. van Aken, T. T. M. Palstra, A. Filippetti, N. A. Spalding, Nature Mater. 3, 164 (2004)

10. K. Lukaszewicz, J. Karut-Kalicínska, Ferroelectrics 7, 81 (1974)

11. B. B. van Aken, A. Meetsma, T. T. M. Palstra, Acta Crystallogr. Sect. C 57, 230 (2001)

12. J. E. Medvedeva, V. I. Anisimov, M. A. Korotin, O. N. Mryasov, A. J. Freeman, J. Phys.: Condens. Matter 12, 4947 (2000)

13. M. Qian, J. Dong, Q. Zheng, Phys. Lett. A 270, 96 (2000)

14. C. Zhong, X. Jiang, H. Yu, Q. Jiang, J. Fang, Z. Li, J. Magn. Magn. Mater. 321, 1260 (2009)

15. R. A. de Souza et al., Phys. Rev. B 84, 104416 (2011)

16. J.-H. Kim et al., Phys. Rev. B 78, 245115 (2008)

17. S. Partzsch, S. Wilkins, J. Hill, E. Schierle, E. Weschke, D. Souptel, B. Büchner, J. Geck, Phys. Rev. Lett. 107, 057201 (2011)

18. J. N. Gonçalves, V. S. Amaral, J. G. Correia, A. M. L. Lopes, Phys. Rev. B 83, 104421 (2011)

19. J. N. Gonçalves, A. Stroppa, J. G. Correia, T. Butz, S. Picozzi, A. S. Fenta, V. S. Amaral, Phys. Rev. B 86, 035145 (2012) 